\title{
BMJ Open Surgical trends, outcomes and disparities in minimal invasive surgery for patients with endometrial cancer in England: a retrospective cohort study
}

\author{
Esther L. Moss (D , , ${ }^{1}$ George Morgan, ${ }^{2}$ Antony P. Martin,, ${ }^{2,3}$ Panos Sarhanis, ${ }^{4}$ \\ Thomas Ind ${ }^{5,6}$
}

To cite: Moss EL, Morgan G, Martin AP, et al. Surgical trends, outcomes and disparities in minimal invasive surgery for patients with endometrial cancer in England: a retrospective cohort study. BMJ Open 2020;10:e036222. doi:10.1136/ bmjopen-2019-036222

- Prepublication history and additional material for this paper are available online. To view these files, please visit the journal online (http://dx.doi. org/10.1136/bmjopen-2019036222).

Received 09 December 2019 Revised 01 July 2020 Accepted 03 July 2020

Check for updates

(c) Author(s) (or their employer(s)) 2020. Re-use permitted under CC BY-NC. No commercial re-use. See rights and permissions. Published by BMJ.

${ }^{1}$ Leicester Cancer Research Centre, University of Leicester, Leicester, UK

${ }^{2}$ HCD Economics, Warrington, UK ${ }^{3}$ Molecular and Clinical Pharmacology, University of Liverpool, Liverpool, UK ${ }^{4}$ Department of Gynaecology, North West London Hospitals NHS Trust, Harrow, UK ${ }^{5}$ Department of Gynaecological Oncology, Royal Marsden Hospital NHS Trust, London, UK ${ }^{6}$ Department of Obstetrics and Gynaecology, St George's University of London, London, UK

Correspondence to

Dr Esther L. Moss

em321@le.ac.uk

\section{ABSTRACT}

Objective To examine surgical outcomes and trends in the implementation of minimally invasive surgery (MIS) use for endometrial cancer (EC).

Design Retrospective cohort study.

Setting English National Health Service hospitals 20112017/2018.

Population 35304 patients having a hysterectomy for EC identified from Hospital Episode Statistics.

Methods Univariate and multivariate analyses compared MIS to open hysterectomy $(\mathrm{OH})$ by assessing the association between demographic, clinical and hospital characteristics by using logistic regression. A propensity score was created, to control for confounding factors including demographics, clinical and hospital characteristics, from a logistic regression which enabled the inverse probability weighting of treatment to be applied in order to compare outcomes of treatment.

Main outcome measures The association between route of surgery on perioperative morbidity and mortality.

Results The MIS rate rose from $40.3 \%$ in 2011 to $68.7 \%$ in $2017 / 2018$, however, there was significant geographica variation $(p<0.001)$. The overall 90 -day mortality was significantly higher with $\mathrm{OH}$ versus MIS (OR $0.34,95 \% \mathrm{Cl}$ 0.18 to $0.62, p=0.0002)$. MIS rates were significantly lower in patients from the lowest socioeconomic group (LSEG) compared with patients from the highest group (HSEG) $(55.4 \%$ vs $59.9 \%, p<0.01)$, and in the black population as compared with white and Asian populations ( $40.4 \%$ vs $58.6 \%$ and $56.0 \%, p<0.0001)$. When patients from LSEG and black patients were treated in hospitals with high MIS rates, the MIS rate increased close to that of the HSEG and white patients $(81.0 \%$ and $74.1 \%$ vs $83.2 \%$ and $82.6 \%$ ).

Conclusions Further investigation is needed to understand the barriers to MIS and improve access so that as many patients as possible can benefit from the reduced morbidity/mortality associated with MIS.

\section{INTRODUCTION}

There are estimated to be 382069 new cases of endometrial cancer (EC) and 89929 deaths worldwide in 2018. ${ }^{1}$ The incidence of EC in the UK is rising, ${ }^{2}$ attributed due to increasing rates of obesity, an ageing
Strengths and limitations of this study

- The main strength of this study is that it is population based and the large number of procedures has enabled trends to be identified that may not have been seen in smaller datasets.

- Hospital Episode Statistics (HES) data only covers National Health Service (NHS)-funded care in England, therefore, data on hysterectomies undertaken outside of the NHS were not included in this study.

- The reliability of the coding might have changed over time although there was no evidence of changes in treatment coding or significant changes in the underlying study population.

- HES database reliably captures extensive amount of demographic, diagnosis and procedure outcomes, however, there is a lack of cancer stage information therefore it is possible some of the deaths may have been due to disease progression rather as a result of surgery.

- Not all deaths are captured in the HES database, only those which occurred in hospital which limits the death data captured in the HES database.

population and falling hysterectomy rates for benign disease. ${ }^{3}{ }^{4}$ Surgery remains the primary treatment option, ${ }^{5}$ particularly for early-stage EC which is associated with a high overall survival rate. However, a less favourable benefit/risk profile for surgical outcomes exists in an elderly population with multiple comorbidities, where consideration is needed as to whether surgical morbidity and mortality would be higher than the risk of recurrence associated with non-surgical treatment options. ${ }^{5}$

The benefit of minimally invasive surgery (MIS) over traditional open surgery for EC has been firmly established with non-inferiority of survival and recurrence rates, and significantly lower levels of postoperative complications. ${ }^{6-8}$ Initially studies were confined to 
early stage endometrioid cancers, ${ }^{79}$ however, as the MIS skills of gynaecologists have developed enabling more extensive surgery, including pelvic/para-aortic lymph node dissection, this evidence has been extended to highrisk subtypes and more advanced disease. ${ }^{10}$ As a result, MIS is the advocated as the preferred surgical route for the management of endometrial malignancy. ${ }^{11} 12$

The surgical management of EC in the UK is divided between satellite cancer units and central cancer centres, following the recommendations from the Calman Hine report in $1995 .{ }^{13}$ Cancer units typically manage earlystage, low-grade cases and refer complex or higher stage/ grade cases to their affiliated regional cancer centre. The adoption of MIS by the gynaecological oncology community is increasing, particularly for the treatment of EC, ${ }^{14}$ however, national data from the USA in 2016 showed that the open hysterectomy $(\mathrm{OH})$ was still the most common procedure rather than MIS. ${ }^{15}$ Similarly, across the UK the uptake of MIS hysterectomy has been variable and significant regional differences exist. ${ }^{16}$

We performed an analysis using Hospital Episode Statistics (HES) for England. ${ }^{17}$ Our primary objective was to examine trends in the implementation of MIS use in EC across England. Our secondary objectives were to examine predictors of uptake of MIS and to further explore the association between route of surgery on perioperative morbidity and mortality.

\section{METHODS}

\section{Data source}

Data were sourced from the HES database to conduct a retrospective cohort analysis from 2011-2017/2018. ${ }^{17}$ No ethical approval was required for this study. The data were accessed by GM and analysed by GM and AM. Access to the data and HCD economics were funded by Intuitive Surgical. None of the clinicians involved in this study received any funding from Intuitive Surgical for this study. Intuitive Surgical did not have access to the data or involvement with the study design, data analysis and writing of the manuscript.

\section{Cohort selection}

From the HES database, an extracted dataset was created to only include patients that had specific Office of Population Censuses and Surveys Classification of Interventions and Procedures V.4.7 (OPCS-4.7) procedure codes which indicated they had a hysterectomy between the dates of October 2011 and December 2017. The specific OPCS-4.7 codes were: Q07.1, Q07.2, Q07.3, Q07.4, Q07.5, Q07.8, Q07.9, Q08.1, Q08.2, Q08.3, Q08.8 and Q08.9. The cohort of female patients was then restricted to be greater or equal to 18 years of age and further by using International Statistical Classification of Diseases and Related Health Problems, 10th Revision (ICD-10) primary diagnosis codes to identify patients where the indication was endometrial/uterine carcinoma or endometrial carcinoma in situ (ECIS) (complex atypical hyperplasia) (C540, C541, C542, C543, C548, C549, C55X and D070).

To classify the hysterectomy approach in terms of intention to treat, we used additional OPCS- 4.7 codes. To identify laparoscopic hysterectomy (LH) the OPCS-4.7 codes were Y75.1, Y75.2, Y75.5, Y75.8, Y75.9, T43.9 and for robotic hysterectomy (RH) the OPCS-4.7 code Y75.3 was used. As a result, MIS is a combination of all the additional OPCS- 4.7 codes. The remainder that did not have any of the additional OPCS- 4.7 codes were classified based on the original procedure codes for the approaches of $\mathrm{OH}$ (Q07.1, Q07.2, Q07.3, Q07.4, Q07.5, Q07.8 and Q07.9) and vaginal hysterectomy (VH) (Q08.1, Q08.2, Q08.3, Q08.8 and Q08.9). In the cases where the OPCS-4.7 code Y71.4 (Failed minimal access approach converted to open) was recorded, and no MIS approach codes were reported alongside. A method to assign intention to treat as LH until the National Health Service (NHS) providers introduced robotic assisted surgery equipment and then classify intention to treat as robotic thereafter.

\section{Patient characteristics}

Demographic data that were collected included age, which was divided into 6 groups by 10 -year intervals from the age of 50. Ethnicity data were collected and patients were grouped into Asian, black, other and white ethnicity.

The English Index of Multiple Deprivation (IMD) ${ }^{18}$ for 2015 was collected to inform the socioeconomic deprivation of patients where a higher rank indicated a less deprived group and a lower rank indicated a more deprived group. The IMD score combines seven indicators (income, employment, health deprivation and disability, education, skills and training, barriers to housing and services, crime, and living environment), into a single deprivation index. The IMD ranks were then split into statistical quartiles to indicate whether the socioeconomic status was high (>25083), intermediate (17475-25083), low (9618-17474) or very low (<9618). Based on postcode of residence, each patient who received EC surgery was assigned to a lower super output area (LSOA) and this was mapped to an IMD rank calculated across the whole of England using this measure.

Comorbidities were examined using the Charlson Comorbidity Index $(\mathrm{CCI})^{19}$ and an additional list of other comorbidities were assessed using a list of ICD-10 codes. ${ }^{20}$

Hospital characteristics were evaluated for the region where the procedure took place and was classified into 10 groups (East, East Midlands, Greater London, Home Counties, North East, North West, South East, South West, West Midlands, Yorkshire). The hospital volume was also analysed as an annual mean across years (if more than 1 year was available) of all hysterectomies performed for $\mathrm{EC} / \mathrm{ECIS}$ at the providers and grouped by statistical quartiles; high (>220), intermediate (71-220), low (70-21) and very low (0-20). In addition, each NHS hospital provider was classified by their MIS rate for hysterectomies performed on EC/ECIS and split into four groups: 
very low $0 \%-25 \%$, low $26 \%-50 \%$, intermediate $51 \%-75 \%$, high $76 \%-100 \%$.

\section{Outcomes}

To analyse the trend of surgeries the number of procedures by each approach was assessed each year to see how practice had changed. The key surgical outcomes of interest were mortality and complications associated with each approach following the procedure. A list of complications were collated (ICD-10/OPCS-4.7 codes) for complication groups of gastrointestinal, wounds, infections, renal/genitourinary and endocrine, cardiovascular, pulmonary, neurological, haemorrhage, urinary tract, ureteric and other. These were assessed as perioperative (within the intervention admission) and at 90 days following intervention to ensure a long enough follow-up period to be assessed after surgery. ${ }^{21}$ Perioperative outcomes included mortality, conversion to $\mathrm{OH}$, recorded complications and length of stay. The 90-day outcomes also included in-hospital mortality and reported complications following the intervention. To assess disparities outcomes were assessed by regional variations at the patient level and additionally the provider level. Subgroup analysis was undertaken to compare the outcomes of high versus low socioeconomic groups and black ethnicity versus other ethnicities.

\section{Statistical analyses}

A descriptive analysis of patient characteristics and outcomes was performed. The different approaches (LH, $\mathrm{RH}, \mathrm{OH}$ and MIS) were then compared by using t-test (for independent samples) and Wilcoxon rank-sum test (Mann-Whitney U test) for continuous variable and for categorical variables by using the $\chi^{2}$ tests. Univariate and multivariate analyses compared MIS to $\mathrm{OH}$ and $\mathrm{RH}$ to $\mathrm{OH}$ by assessing the association between demographic, clinical and hospital characteristics by using a logistic regression. Based on the predicted probability, a propensity score was created from the logistic regression which included year of surgery, age groups, ethnicity, IMD rank groups, CCI group, region and provider volume. The predicted probabilities enabled the inverse probability weighting of treatment (IPTW) to be applied to balance observed confounders between the treatments. ${ }^{22}$ To ensure balance of the measured covariate distribution between treatment groups, the standardised mean difference was assessed using a threshold for mean difference of $0.1 .^{23}$ Application of the IPTW was then used on comparing outcomes of treatment. Missing data of any of the propensity score variables were not imputed and patients with missing data were removed from propensity score matching. All descriptive and regression analyses were performed using Stata V.15.

\section{Patient and public involvement}

There was no patient or public involvement in the study planning or design.

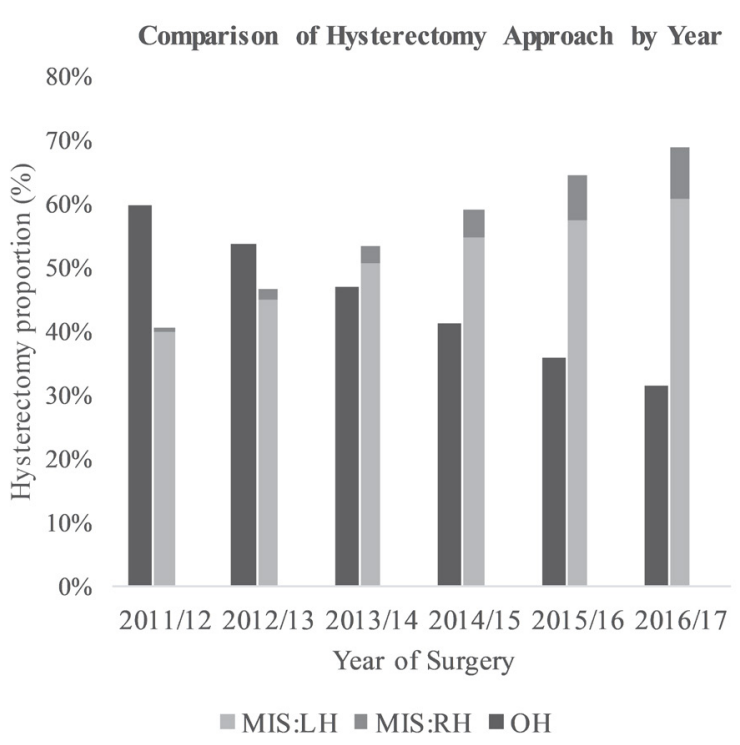

Figure 1 Route of hysterectomy by year. The route of surgery for endometrial cancer over time. LH, laparoscopic hysterectomy; MIS, minimally invasive surgery; $\mathrm{OH}$, open hysterectomy; $\mathrm{RH}$, robotic hysterectomy.

\section{RESULTS}

A total of 35304 procedures were performed, 20405 (57.8\%) were MIS (LH 18604 and RH 1801), 14291 (40.5\%) OH and 608 (1.7\%) VH. Due to the low numbers the $\mathrm{VH}$ cases were not included in the analysis. The rate of MIS rose from $40.3 \%$ to $68.7 \%$ across the study period ( $\mathrm{p}<0.001$ ), with RH increasing from $0.7 \%$ in $2011 / 12 \%$ to $8.2 \%$ in $2016 / 17(\mathrm{p}<0.001)$, whereas the rate of $\mathrm{OH}$ fell from $59.7 \%$ in $2011 / 12$ to $31.3 \%$ in $2016 / 17$ ( $<<0.001$ ) (figure 1). Lymph node dissection was performed in $18.4 \%$ of $\mathrm{OH}$ and $15.6 \%$ of MIS (14.6\% LH, $25.3 \% \mathrm{RH})$ cases.

Table 1 presents the unadjusted and IPTW patient characteristics of those that received MIS or OH. Comparison of the unadjusted results of MIS and $\mathrm{OH}$ cases on patient characteristics identified that the $\mathrm{OH}$ group contained patients who were on average younger ( 66.1 vs 65.7 years, $\mathrm{p}=0.0052)$, of non-white ethnicity $(\mathrm{p}<0.001)$ and on average from a lower socioeconomic group (17483 vs 16 $788, \mathrm{p}<0.001)$. There was no difference in the levels of obesity $(24.0 \%$ vs $23.6 \%, \mathrm{p}=0.504)$ between MIS and $\mathrm{OH}$, however, there was a difference in the CCI score, (1.41 vs $1.52, \mathrm{p}<0.001)$.

This was confirmed in the multivariate regression which found that a patient was more likely to receive MIS if the surgery was performed more recently, they were aged between 50 and 90 years and had the procedure undertaken in the North East or South West of England ( $<<0.001$ for all: online supplementary appendix 1 ). Whereas a patient was more likely to receive $\mathrm{OH}$ if they attended a very low volume centre, were from a very low socioeconomic group, were of black or Other ethnicity, and located in the North West or West Midlands of England $(\mathrm{p}<0.001$ for all: online supplementary appendix 2$)$. 
Table 1 Clinical and demographic characteristics by the cohorts of hysterectomy approach

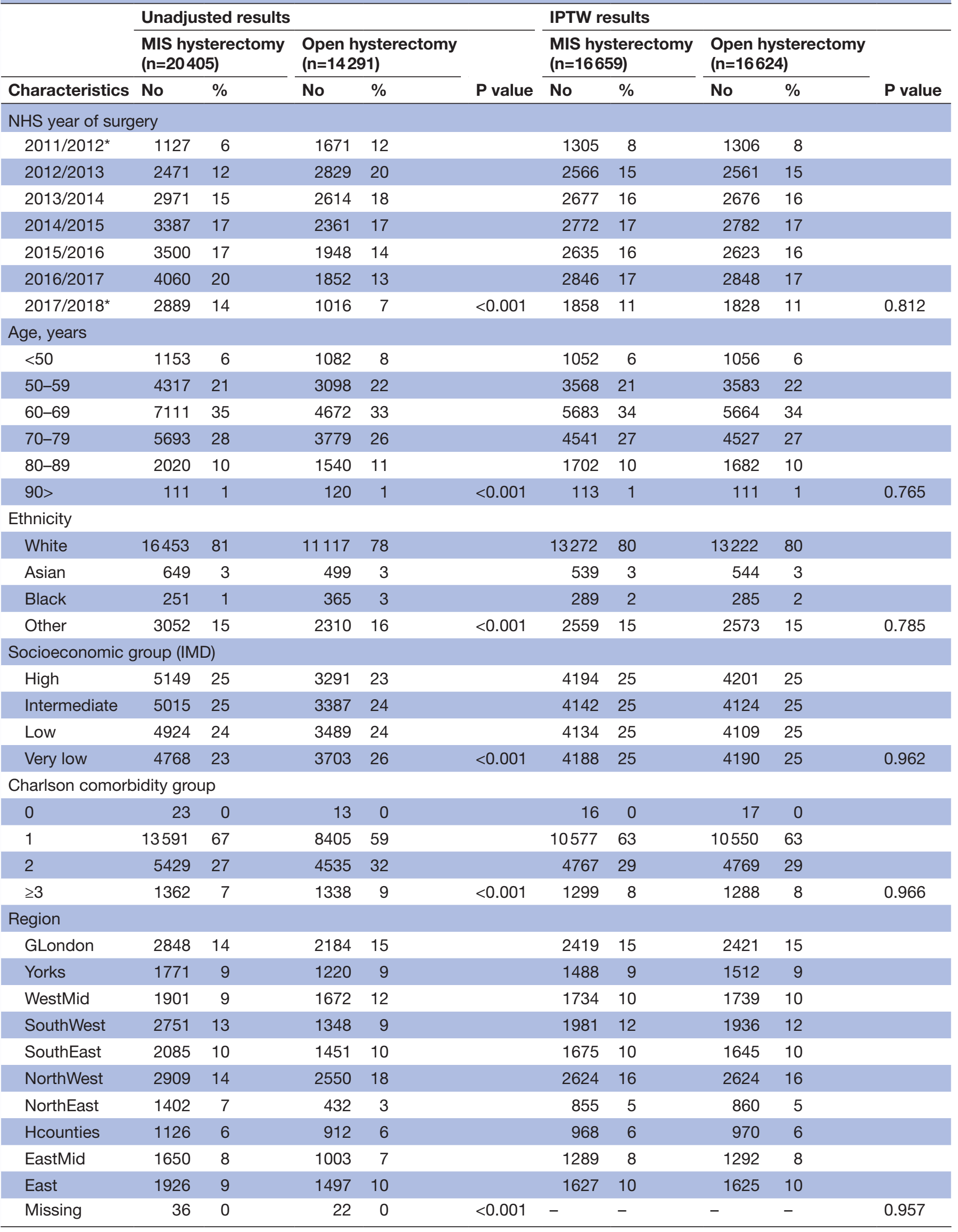


Table 1 Continued

\begin{tabular}{|c|c|c|c|c|c|c|c|c|c|c|}
\hline \multirow[b]{3}{*}{ Characteristics } & \multicolumn{5}{|c|}{ Unadjusted results } & \multicolumn{5}{|c|}{ IPTW results } \\
\hline & \multicolumn{2}{|c|}{$\begin{array}{l}\text { MIS hysterectomy } \\
(\mathrm{n}=20405)\end{array}$} & \multicolumn{2}{|c|}{$\begin{array}{l}\text { Open hysterectomy } \\
(n=14291)\end{array}$} & \multirow[b]{2}{*}{$P$ value } & \multicolumn{2}{|c|}{$\begin{array}{l}\text { MIS hysterectomy } \\
(n=16659)\end{array}$} & \multicolumn{2}{|c|}{$\begin{array}{l}\text { Open hysterectomy } \\
(\mathrm{n}=16624)\end{array}$} & \multirow[b]{2}{*}{$P$ value } \\
\hline & No & $\%$ & No & $\%$ & & No & $\%$ & No & $\%$ & \\
\hline \multicolumn{11}{|l|}{ Provider volume } \\
\hline High & 12725 & 62 & 8703 & 61 & & 10445 & 63 & 10456 & 63 & \\
\hline Intermedate & 7140 & 35 & 5102 & 36 & & 5937 & 36 & 5894 & 35 & \\
\hline Low & 288 & 1 & 191 & 1 & & 232 & 1 & 231 & 1 & \\
\hline Very low & 36 & 0 & 58 & 0 & & 46 & 0 & 43 & 0 & \\
\hline Missing & 216 & 1 & 237 & 2 & $<0.001$ & - & - & - & - & 0.712 \\
\hline
\end{tabular}

${ }^{*}$ NHS year 2011/2012 and 2017/2018 not full year.

IMD, Index of Multiple Deprivation; IPTW, inverse probability weighting of treatment; MIS, minimally invasive surgery; NHS, National Health

Service.

\section{Surgical morbidity and mortality}

A significant difference was seen in the perioperative outcome of the different routes of surgery. The length of hospital stay was significantly longer for $\mathrm{OH}$ as compared with MIS (5.28 vs 2.32 days, $\mathrm{p}<0.001$ ). The overall conversion rate to OH for MIS was 6.6\%. IPW analysis identified that the only peri-operative complication that was not significantly higher with $\mathrm{OH}$ as compared with MIS was ureteric complications $(\mathrm{p}<0.001$ for all other complications: table 2). The surgical complications requiring readmission/reintervention in 90 days were also significantly higher $(9.6 \%$ vs $5.5 \%, \mathrm{p}<0.001)$ in the $\mathrm{OH}$ cohort.
The overall mortality up to 90 days after surgery following IPTW was significantly higher with $\mathrm{OH}$ rather than MIS (OR $0.34 ; 95 \%$ CI 0.18 to $0.62 ; \mathrm{p}=0.0002$ : table 2). This relationship was accentuated when comparing RH versus $\mathrm{OH}$ (OR 0.04; $95 \%$ CI 0 to 0.27 ; $\mathrm{p}<0.001)$. The patients who died in the 90-day period following surgery were older (70.6 vs 70.4 years, $\mathrm{p}=0.0012)$ and had more comorbidities, in particular diabetes $(\mathrm{p}=0.024)$ hypertension $(\mathrm{p}=0.018)$, renal failure $(\mathrm{p}=0.001)$ and angina $(\mathrm{p}=0.017)$, compared with patients who did not die.

Table 2 Comparison of mortality and complications at intervention between MIS and OH cohorts

\begin{tabular}{|c|c|c|c|c|c|c|c|c|c|c|c|c|}
\hline & \multicolumn{6}{|c|}{ Unadjusted results } & \multicolumn{6}{|c|}{ IPTW results } \\
\hline & \multicolumn{2}{|l|}{ MIS } & \multicolumn{2}{|l|}{$\mathrm{OH}$} & \multirow[b]{2}{*}{$P$ value } & \multirow{2}{*}{$\begin{array}{l}\text { MIS versus OH } \\
\text { OR }(95 \% \mathrm{Cl})\end{array}$} & \multicolumn{2}{|l|}{ MIS } & \multicolumn{2}{|l|}{$\mathrm{OH}$} & \multirow[b]{2}{*}{$P$ value } & \multirow{2}{*}{$\begin{array}{l}\text { MIS versus OH } \\
\text { OR }(95 \% \mathrm{Cl})\end{array}$} \\
\hline & No & $\%$ & No & $\%$ & & & No & $\%$ & No & $\%$ & & \\
\hline $\begin{array}{l}\text { Death } \\
\text { (intervention) }\end{array}$ & 9 & 0 & 18 & 0 & 0.007 & 0.35 (0.14 to 0.82$)$ & 8 & 0 & 25 & 0 & 0.003 & $0.32(0.12$ to 0.73$)$ \\
\hline Death (overall)* & 17 & 0 & 44 & 0 & $<0.001$ & 0.27 (0.14 to 0.48$)$ & 15 & 0 & 44 & 0 & $<0.001$ & 0.34 (0.18 to 0.62$)$ \\
\hline $\begin{array}{l}\text { Any complication } \\
\text { (intervention) }\end{array}$ & 4305 & 21 & 4012 & 28 & 01 & 0.54 (0.51 to 0.57$)$ & 3503 & 21 & 4725 & 28 & 01 & $0.67(0.64$ to 0.71$)$ \\
\hline \multicolumn{13}{|c|}{ Specific complications (intervention) } \\
\hline Gastrointestinal & 1637 & 8 & 1185 & 8 & $<0.001$ & 0.61 (0.57 to 0.66$)$ & 1275 & 8 & 1479 & 9 & $<0.001$ & 0.85 (0.79 to 0.92$)$ \\
\hline Wounds & 325 & 2 & 679 & 5 & $<0.001$ & 0.20 (0.17 to 0.23$)$ & 276 & 2 & 783 & 5 & $<0.001$ & $0.34(0.30$ to 0.39$)$ \\
\hline Infections & 360 & 2 & 815 & 6 & $<0.001$ & $0.16(0.14$ to 0.19$)$ & 296 & 2 & 913 & 5 & $<0.001$ & $0.31(0.27$ to 0.36$)$ \\
\hline Urinary & 623 & 3 & 786 & 5 & $<0.001$ & $0.26(0.24$ to 0.29$)$ & 514 & 3 & 899 & 5 & $<0.001$ & 0.56 (0.50 to 0.62$)$ \\
\hline Ureteric & 6 & 0 & 8 & 0 & 0.005 & 0.25 (0.07 to 0.82$)$ & 4 & 0 & 8 & 0 & 0.247 & 0.50 (0.11 to 1.86$)$ \\
\hline Cardiovascular & 1255 & 6 & 1162 & 8 & $<0.001$ & 0.30 (0.27 to 0.32$)$ & 1047 & 6 & 1340 & 8 & $<0.001$ & 0.77 (0.70 to 0.83 ) \\
\hline Pulmonary & 305 & 1 & 587 & 4 & $<0.001$ & $0.13(0.11$ to 0.15$)$ & 262 & 2 & 676 & 4 & $<0.001$ & $0.38(0.33$ to 0.44$)$ \\
\hline Neurological & 84 & 0 & 111 & 1 & $<0.001$ & 0.18 (0.14 to 0.24$)$ & 77 & 0 & 120 & 1 & 0.002 & 0.64 (0.47 to 0.86$)$ \\
\hline Haemorrhage & 199 & 1 & 270 & 2 & $<0.001$ & $0.17(0.14$ to 0.20$)$ & 169 & 1 & 310 & 2 & $<0.001$ & $0.54(0.44$ to 0.65$)$ \\
\hline Other & 736 & 4 & 646 & 5 & $<0.001$ & $0.22(0.20$ to 0.25$)$ & 593 & 4 & 786 & 5 & $<0.001$ & 0.74 (0.67 to 0.83$)$ \\
\hline
\end{tabular}

*Death (overall) Includes death at intervention and deaths reported 90 days after intervention.

IPTW, inverse probability weighting of treatment; MIS, minimally invasive surgery; $\mathrm{OH}$, open hysterectomy. 


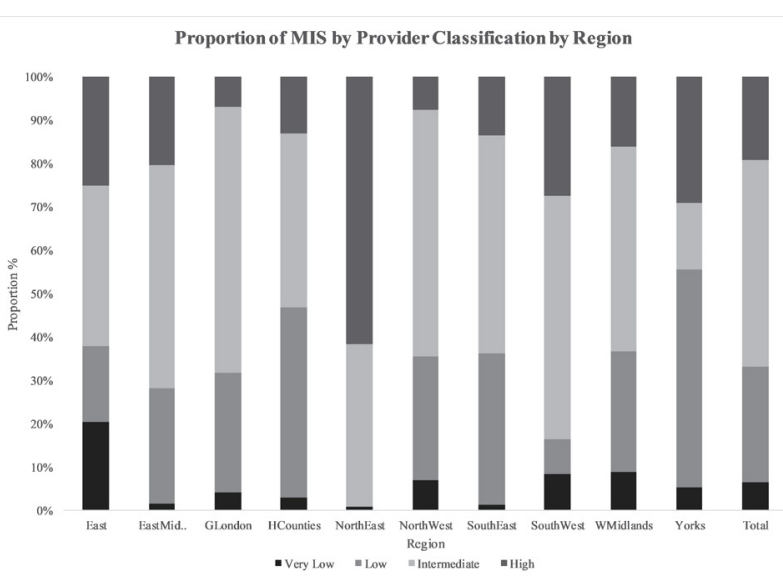

Figure 2 Providers MIS rate classification by region. The proportion, by region, of providers classified as having high, intermediate, low and very low MIS rates (very low 0\%$25 \%$ MIS rate, low 26\%-50\% MIS rate, intermediate $51 \%-$ $75 \%$ MIS rate, high $76 \%-100 \%$ MIS rate). MIS, minimally invasive surgery.

\section{Regional variation}

The MIS rate increased year on year across all regions, however, the uptake varied significantly between geographical regions $(p<0.001$; table 1$)$. Very low volume centres $(<20$ cases per year) had a significantly lower MIS rate compared with the other quartiles, $37.5 \%$ vs $58.9 \%$ for low, $56.7 \%$ intermediate and $58.9 \%$ for high, $(\mathrm{p}<0.05$ for all). Provider level analysis showed only 55 centres had high MIS levels for EC ( $>76.0 \%$ MIS rate) and there was a large disparity between regions (figure 2). Over time the age of the patients undergoing MIS increased and by 2017 was the median age was higher than for $\mathrm{OH}$ cases, with the greatest increase being seen in the high MIS providers. The CCI of patients undergoing surgery (any route) between 2011 and 2018 also increased year on year, however, at high and intermediate MIS centres the ratio between CCI of $\mathrm{OH}$ and MIS patients became smaller (1.06 in 2011 to 1.02 in 2017). In addition, there was great variability across providers and regions as to the proportion of MIS implemented, with $78 \%$ of providers achieving a $50 \%$ threshold but only $56 \%$ passing a $70 \%$ threshold (figure 3).

\section{Disparity in MIS rates}

Disparity was seen in the MIS rates in patients from the lowest socioeconomic group as compared with patients from the highest group (55.4\% compared with 59.9\%, $\mathrm{p}<0.01)$. Patients from this group were younger compared with patients in the high socioeconomic group(64.3 vs 67.0 years, $\mathrm{p}<0.01)$ but had a higher level of comorbidities $(1.56$ vs $1.39, \mathrm{p}<0.01)$. When patients from the lowest socioeconomic group were treated in high MIS centres the MIS rate increased to $81.0 \%$, close to the rate in patients from the highest socioeconomic group $83.2 \%$.

Black patients undergoing surgery were significantly younger than patients from other ethnic groups (63.7 vs $66.0, \mathrm{p}<0.001$ ), were mainly located in more deprived

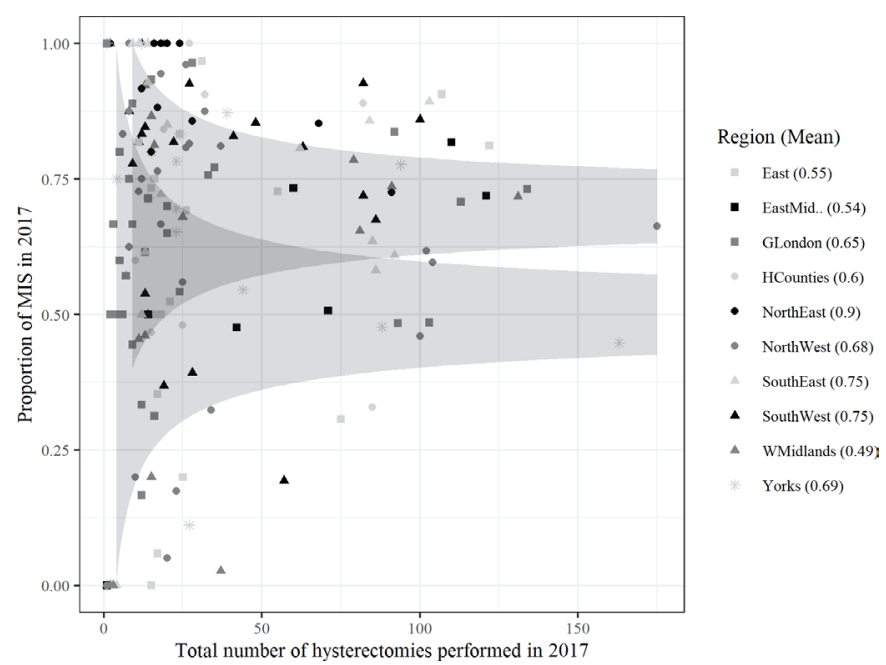

Figure 3 Funnel plot of MIS rate and number of cases performed for 2017. The proportion of hysterectomies performed by MIS by the total number of hysterectomies for each provider that performed at least one hysterectomy in 2017. Each provider is also classified by their region and an average value for each region is plotted. two thresholds are for $50 \%$ and $70 \%$ MIS rate. MIS, minimally invasive surgery.

areas $(85.9 \%$ in low or very low socioeconomic status) and had more comorbidities as compared with the overall population cohort $(1.65$ vs $1.45, \mathrm{p}<0.001)$. The MIS rates were significantly lower in the black population as compared with the white and Asian populations, $40.4 \%$ as compared with $58.6 \%$ and $56.0 \%$, respectively $(\mathrm{p}<0.001)$. A significantly higher percentage of white patients $(21.2 \%)$ attended high MIS centres as compared with Asian or black patients $(7.8 \%$ and $4.7 \%$, respectively), however, when black patients underwent surgery at a high MIS provider, their MIS rate increased to $74.1 \%$ (figure 4).

\section{DISCUSSION}

\section{Main findings}

We have identified significant disparities in the rates of MIS in England forpatients undergoing hysterectomy in the NHS for EC/ECIS. Patients from the lowest socioeconomic group and of non-white ethnicity and were significantly less likely to undergo MIS as compared with white patients from more socially affluent areas. The difference in MIS rates was reduced when patients underwent surgery at high MIS providers, indicating that patient's geographical location and local facilities to provide MIS is a factor in route of surgery for EC.

There are confounding factors that could help to explain the difference between the populations. The CCI score was significantly higher in the lower socioeconomic and black populations, which could have resulted in them being not suitable for MIS. Black ethnicity is known to be associated with a higher incidence and size of uterine fibroids as compared with white women ${ }^{25}$ which again could have contribute to a higher $\mathrm{OH}$ rate by making MIS 


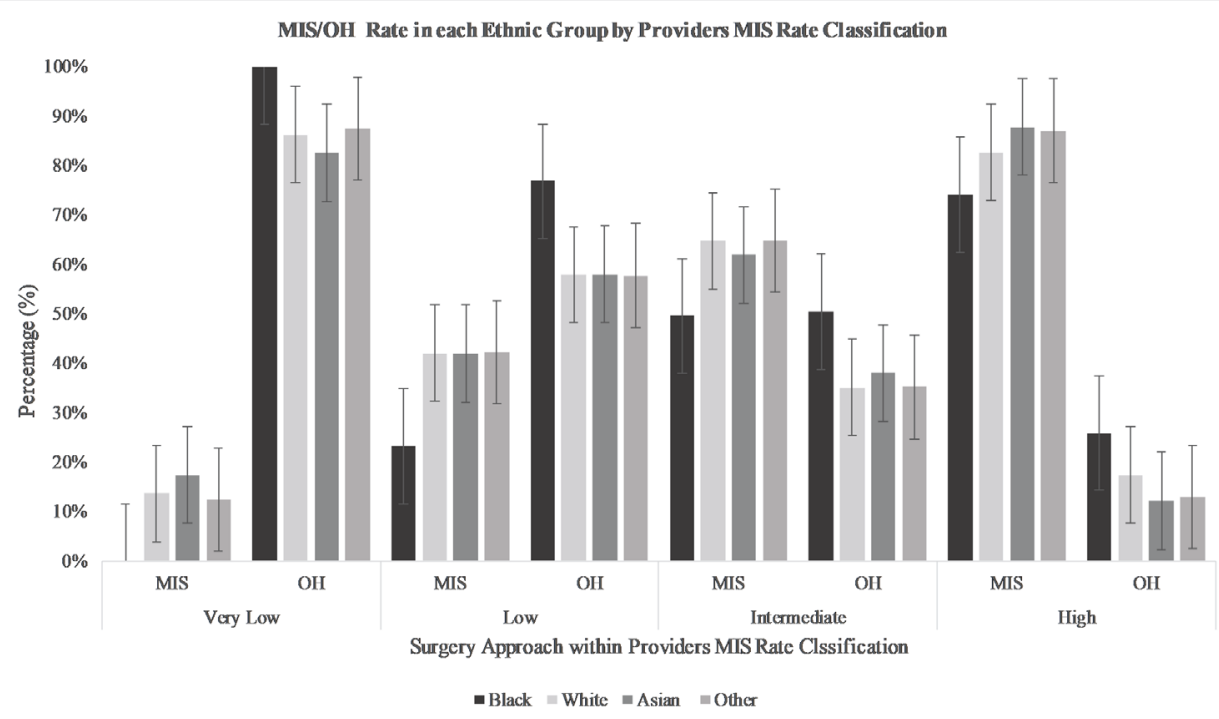

Figure $4 \mathrm{MIS} / \mathrm{OH}$ rate at patient ethnicity by providers MIS rate classification. Comparison of the percentage split between MIS and $\mathrm{OH}$ at each of the MIS rate classifications by patient ethnicity (very low 0\%-25\% MIS rate, low $26 \%-50 \%$ MIS rate, intermediate $51 \%-75 \%$ MIS rate, high 76\%-100\% MIS rate). MIS, minimally invasive surgery; OH, open hysterectomy.

not technically feasible, and non-white ethnicity has been identified as a risk factor for conversion to open surgery. ${ }^{26}$ The previous abdominal surgery rate is also not well recorded in the HES data, therefore, patients who have undergone extensive surgery previously would also not have been appropriate for MIS. Similarly, the presence of fibroids that would preclude MIS is not recorded on HES and would depend on the size/location of the fibroid as well as the experience of the surgical team in performing MIS. However, if these were the only reasons for the disparity it does not explain why the MIS rate increases in patients from the lowest socioeconomic group or of black ethnicity undergoing surgery at high MIS providers.

Unlike in cervical cancer, ${ }^{27} 28$ there has not been shown to be a difference in long-term oncological outcome of the use of MIS as compared with OH in EC. ${ }^{7}$ The perioperative death rate associated with $\mathrm{OH}$ is known to be significantly higher as compared with MIS, however, we have shown the magnitude of the difference is much greater than previously reported. Wright $e t a l$, in his analysis of 6304 patients from the SEER database (2006-2011) identified an OR of death by 30 days for $\mathrm{OH}$ as compared with MIS of 0.57 (95\% CI 0.34 to 0.95$),{ }^{22}$ whereas in our study a threefold increase risk of death was found with OH compared with MIS (OR 0.34; $95 \%$ CI 0.18 to 0.62 ).

The patients who died following surgery were older and more comorbid than the population that survived and as such may have been deemed unsuitable for MIS, although this could only be determined on an individual case by case basis. The age of patients undergoing MIS has increased across all regions since 2012 indicating that surgical teams have gained experience and are aware of the benefits and evidence supporting the use of MIS in the elderly population. ${ }^{29}$ Patient comorbidities, as determined by CCI, do however appear to influence MIS rates, with only the high/intermediate MIS providers demonstrating a rise in the CCI level of their cases over time, suggesting that one reason why there is such a high proportion of MIS cases in some centres is because they undertake cases MIS that other centres may deem unsuitable or too high risk for MIS. This highlights the challenges of operating on patients with medical comorbidities since surgical risk is not confined to the technicalities of the surgery but encompasses the patient's entire perioperative care, including preoperative optimisation, anaesthetic procedure and postoperative mobilisation, nutrition and recovery. In particular, the anaesthetic experience and high-dependency unit support to undertake challenging cases MIS may be lacking in hospitals only performing a small number of EC/ECIS cases per year, which may in turn contribute to the significantly lower MIS rates in these centres. ${ }^{30}$

Sharing expertise and providing support and mentorship for low MIS providers through local cancer centre/ unit networks could help increase the overall MIS rates, however, the introduction of a national best practice target in England may help to focus resources on this population so that patients from all backgrounds and areas can benefit. MIS rates for EC have been proposed a quality indicator, ${ }^{31}$ and the target set in Scotland has been raised from $50 \%$ in 2014 to $70 \%$ in 2018, reflecting the change in clinical practice. ${ }^{32}$ We have shown that in 2017 only $56 \%$ of providers reached the $70 \%$ threshold, therefore, much greater support for gynaecologists and their surgical teams will be needed in order for the majority of providers in England to achieve this target. Although the 95\% MIS rate achieved in Denmark ${ }^{33}$ would suggest that this target could be set even higher, thereby impacting on the significantly higher mortality seen with $\mathrm{OH}$, and act as an incentive for individual providers to analyse their cases and strive to increase their proportion of MIS procedures further. If improvements cannot be made in the low 
MIS rate seen in some of the very low volume providers it does raise the argument as to whether a minimum annual caseload should be introduced for hospitals, with further centralisation of EC surgical management to larger providers, in order to increase access to MIS. Referral to larger providers may impact on overall MIS rates, however, it could result in greater disruption and burden to the patient rather than being treated by their local provider since, as already discussed, this population may be elderly and comorbid.

\section{Strengths and limitations}

The main strength of this study is that it is population based and the large number of procedures has enabled trends to be identified that may not have been seen in smaller datasets. It should be noted that HES data only covers NHS-funded care in England, therefore, data on hysterectomies undertaken outside of the NHS were not included in this study. Despite its limitations, in particular the lack of cancer stage information and potential recording or adjudication errors, the ethnicity data in HES data are known to be reliable and match the UK population. ${ }^{32}$ The lack of staging data means that it is not possible to identify the small proportion of cases that would have undergone more extensive open cytoreductive surgery, which would be associated with a substantially higher morbidity/mortality rate than an MIS staging procedure. Cytoreductive procedures would only have taken place at designated cancer centres due to the location of specialist gynaecological oncology surgeons, and therefore, such procedures will not have impacted the rate of open surgery or outcomes from cancer units. Despite a lack of stage data the pelvic lymphadenectomy rate is known and has been included, which was comparable across the groups $(18.4 \%$ in $\mathrm{OH}, 14.6 \%$ in $\mathrm{LH}$ and $25.3 \%$ in $\mathrm{RH}$ ).

The reliability of the coding might have changed over time although there was no evidence of changes in treatment coding or significant changes in the underlying study population. Another limitation relates to the sensitivity of IMD quintiles matched to each LSOA. Moreover, within each LSOA, there may be significant variation in terms of deprivation and therefore future research should assess deprivation at an individual level

Confining the survival analysis to 90 days following surgery aimed to capture procedure related rather than disease-specific deaths, although since stage data are not available, it is possible some of the deaths may have been due to disease progression rather as a result of surgery.

\section{Interpretation}

Disparities exist in the use of MIS for the treatment of EC/ECIS in England. Further investigation of patientlevel data, preference elicitation studies and qualitative research are needed to understand the potential barriers to MIS in clinical practice and to improve access so that as many patients as possible can benefit from the reduced morbidity and mortality associated with MIS.
Acknowledgements We would like to acknowledge the contributions from Thomas Burke, Gabriel Pedra and from HCD Economics who assisted in the conduct of this study. We would like to thank Harvey Walsh for facilitating access to Hospital Episode Statistics Data via a Standard Extract Re-use Agreement issued by the Health and Social Care Information Centre 2018.

Contributors ELM, TI, GM and AM contributed to the conceptualisation and study design. Data analysis and interpretation was performed by ELM, TI, GM, AM and PS. Figures and tables creation was performed by ELM, GM and AM. All authors were involved in the writing or review of the manuscript and approved the final version.

Funding HCD economics were funded by Intuitive Surgical. None of the clinicians involved in this study received funding from Intuitive Surgical.

Disclaimer Intuitive Surgical did not have any involvement with the study design, data analysis and writing of the manuscript.

Competing interests ELM and TI perform Da'Vinci robotic gynaecological surgery (Intuitive Surgical) and are members of the British and Irish Association of Robotic Surgeons (BIARGS), which is supported by Intuitive Surgical and other robotics/ laparoscopic companies to hold education/training events. ELM has been awarded research grants from Intuitive Surgical and Hope Against Cancer for unrelated studies. TI has done two days paid consultancy work for Medtronic. GM and AM from HCD Economics were funded by Intuitive Surgical.

Patient consent for publication Not required.

Ethics approval The HES database is managed by NHS Digital and is available for research without ethical approval.

Provenance and peer review Not commissioned; externally peer reviewed.

Data availability statement Data may be obtained from a third party and are not publicly available. Data analysed in this study is available through Hospital Episode Statistics (HES).

Open access This is an open access article distributed in accordance with the Creative Commons Attribution Non Commercial (CC BY-NC 4.0) license, which permits others to distribute, remix, adapt, build upon this work non-commercially, and license their derivative works on different terms, provided the original work is properly cited, appropriate credit is given, any changes made indicated, and the use is non-commercial. See: http://creativecommons.org/licenses/by-nc/4.0/.

ORCID iD

Esther L. Moss http://orcid.org/0000-0002-2650-0172

\section{REFERENCES}

1 Bray F, Ferlay J, Soerjomataram I, et al. Global cancer statistics 2018: GLOBOCAN estimates of incidence and mortality worldwide for 36 cancers in 185 countries. CA Cancer J Clin 2018:68:394-424.

2 Cancer Research UK. Uterine cancer statistics / cancer research UK. Available: https://www.cancerresearchuk.org/health-professional/ cancer-statistics/statistics-by-cancer-type/uterine-cancer [Accessed 21 Aug 2019].

3 Crosbie E, Morrison J. The emerging epidemic of endometrial cancer: time to take action. In: Tovey D, ed. Cochrane database of systematic reviews. Chichester, UK: John Wiley \& Sons, Ltd, 2014.

4 Reid PC, Mukri F. Trends in number of hysterectomies performed in England for menorrhagia: examination of health episode statistics, 1989 to 2002-3. BMJ 2005;330:938-9.

5 Podzielinski I, Randall ME, Breheny PJ, et al. Primary radiation therapy for medically inoperable patients with clinical stage I and II endometrial carcinoma. Gynecol Oncol 2012;124:36-41.

6 Asher R, Obermair A, Janda M, et al. Disease-free and survival outcomes for total laparoscopic hysterectomy compared with total abdominal hysterectomy in early-stage endometrial carcinoma: a meta-analysis. Int J Gynecol Cancer 2018;28:529-38.

7 Galaal K, Bryant A, Fisher AD, et al. Laparoscopy versus laparotomy for the management of early stage endometrial cancer. Cochrane Database Syst Rev 2012;9:CD006655.

8 Walker JL, Piedmonte MR, Spirtos NM, et al. Laparoscopy compared with laparotomy for comprehensive surgical staging of uterine cancer: gynecologic oncology group study LAP2. J Clin Oncol 2009;27:5331-6.

9 Janda M, Gebski V, Brand A, et al. Quality of life after total laparoscopic hysterectomy versus total abdominal hysterectomy for stage I endometrial cancer (LACE): a randomised trial. Lancet Oncol 2010;11:772-80. 
10 Nieto VL, Huang Y, Hou JY, et al. Use and outcomes of minimally invasive hysterectomy for women with nonendometrioid endometrial cancers. Am J Obstet Gynecol 2018;219:463.e1-463.e12.

11 Sundar S, Balega J, Crosbie E, et al. BGCS uterine cancer guidelines: recommendations for practice. Eur J Obstet Gynecol Reprod Biol 2017;213:71-97.

12 NICE. Interventional procedure overview of laparoscopic hysterectomy (including laparoscopic total hysterectomy and laparoscopically assisted vaginal hysterectomy) for endometrial cancer, 2010. Available: https://www.nice.org.uk/guidance/ipg356/ documents/laparoscopic-hysterectomy-including-laparoscopictotal-hysterectomy-and-laparoscopic-assisted-vaginalhysterectomy-for-endometrial-cancer-overview2 [Accessed 21 Aug 2019].

13 Whitehouse M. A policy framework for commissioning cancer services. BMJ 1995;310:1425-6.

14 Mabrouk M, Frumovitz M, Greer M, et al. Trends in laparoscopic and robotic surgery among gynecologic oncologists: a survey update. Gynecol Oncol 2009;112:501-5.

15 Mannschreck D, Matsuno RK, Moriarty JP, et al. Disparities in surgical care among women with endometrial cancer. Obstet Gynecol 2016;128:526-34.

16 Madhvani K, Curnow T, Carpenter T. Route of hysterectomy: a retrospective, cohort study in English NHS hospitals from 2011 to 2017. BJOG 2019;126:795-802.

17 NHS Digital. Hospital episode statistics (HES). Available: https:// digital.nhs.uk/data-and-information/data-tools-and-services/dataservices/hospital-episode-statistics [Accessed 6 Aug 2019].

18 Government $\mathrm{D}$ for $\mathrm{C}$ and $\mathrm{L}$. The English index of multiple deprivation (IMD) 2015-Guidance, 2015. Available: https://www.gov.uk/ government/statistics/english-indices-of-deprivation-2015 [Accessed 22 Feb 2019].

19 Quan H, Li B, Couris CM, et al. Updating and validating the Charlson comorbidity index and score for risk adjustment in hospital discharge Abstracts using data from 6 countries. Am J Epidemiol 2011;173:676-82.

20 Ma C, Crespin M, Proulx M-C, et al. Postoperative complications following colectomy for ulcerative colitis: a validation study. BMC Gastroenterol 2012;12:39.
21 Adam MA, Turner MC, Sun Z, et al. The appropriateness of 30-day mortality as a quality metric in colorectal cancer surgery. Am J Surg 2018;215:66-70.

22 Wright JD, Burke WM, Tergas Al, et al. Comparative effectiveness of minimally invasive hysterectomy for endometrial cancer. J Clin Oncol 2016;34:1087-96.

23 Austin PC. Balance diagnostics for comparing the distribution of baseline covariates between treatment groups in propensity-score matched samples. Stat Med 2009;28:3083-107.

24 Zhang Z, Kim HJ, Lonjon G, et al. Balance diagnostics after propensity score matching. Ann Transl Med 2019;7:16.

25 Kjerulff KH, Langenberg P, Seidman JD, et al. Uterine leiomyomas. Racial differences in severity, symptoms and age at diagnosis. $J$ Reprod Med 1996;41:483-90.

26 Jones N, Fleming ND, Nick AM, et al. Conversion from robotic surgery to laparotomy: a case-control study evaluating risk factors for conversion. Gynecol Oncol 2014;134:238-42.

27 Melamed A, Margul DJ, Chen L, et al. Survival after minimally invasive radical hysterectomy for early-stage cervical cancer. $N$ Engl J Med 2018;379:1905-14.

28 Ramirez PT, Frumovitz M, Pareja R, et al. Minimally invasive versus abdominal radical hysterectomy for cervical cancer. $N$ Engl J Med 2018;379:1895-904.

29 Uccella S, Bonzini M, Palomba S, et al. Laparoscopic vs. open treatment of endometrial cancer in the elderly and very elderly: an age-stratified multicenter study on 1606 women. Gynecol Oncol 2016;141:211-7.

30 Srivastava A, Niranjan A. Secrets of safe laparoscopic surgery: anaesthetic and surgical considerations. J Minim Access Surg 2010;6:91-4.

31 Bonte A-S, Luyckx A, Wyckmans L, et al. Quality indicators for the management of endometrial, cervical and ovarian cancer. Eur J Surg Oncol 2019;45:528-37.

32 Taskforce SC, Scotland HI. Scottish cancer taskforce cutaneous melanoma clinical quality performance indicators: endometrial cancer - 2014, 2016.

33 Jørgensen SL, Mogensen O, Wu C, et al. Nationwide introduction of minimally invasive robotic surgery for early-stage endometrial cancer and its association with severe complications. JAMA Surg 2019;154:530. 